\section{MRS Bulletin Volume Organizers guide technical theme topics for 2019}

\author{
www.mrs.org/bulletin
}

$T_{0}^{\text {he }}$

he MRS Bulletin 2019 Volume Organizers, appointed by MRS President Sean Hearne, will guide the development of theme topics for the 2019 volume year. They are Craig B. Arnold (Princeton University, USA), Claus Daniel (Oak Ridge National Laboratory and The University of Tennessee, Knoxville, USA), Seung Min Jane Han (Korea Advanced Institute of Science and Technology, South Korea), and Gabriel Montaño (Northern Arizona University and Los Alamos National Laboratory, USA).

Craig B. Arnold is a professor of mechanical and aerospace engineering at Princeton University and is the director

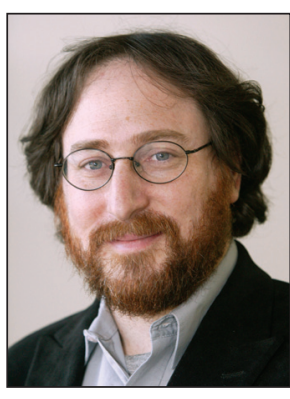

of the Princeton Institute for Science and Technology of Materials. His research ranges from basic science to applied technology, with interests in energystorage systems, laser materials processing, and advanced optics. He earned his $\mathrm{PhD}$ degree from Harvard University, USA, and was a National Research Council postdoctoral fellow at the US Naval Research Laboratory prior to joining the faculty at Princeton in 2003. His work has been recognized by the Office of Naval Research Young Investigator Award and an NSF CAREER Award. He is a Fellow of OSA and SPIE. Arnold volunteered actively for many years on the MRS Public Outreach and Academic Affairs Committees (AAC) in various capacities, including chair of the AAC from 2005 to 2007. In addition, he served as co-chair of a MRS symposium on laser processing of materials and a symposium on oxide thin films for energy applications.

Claus Daniel is the director of sustainable transportation at Oak Ridge National
Laboratory (ORNL) and professor at The University of Tennessee, Knoxville. He manages ORNL's \$90M Sustainable

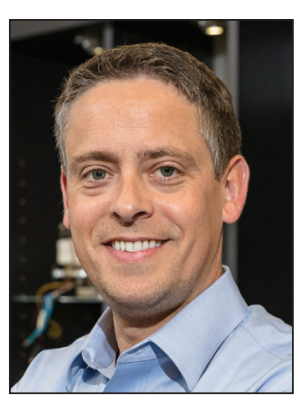

Transportation Program with research foci in electrification, combustion and emission, data science and connected vehicles, and materials for future systems, with a team of approximately 220 people across ORNL. He is a materials scientist by training, with experience in processing, manufacturing, and characterization development for automotive and manufacturing systems. He earned two MS degrees: one from Saarland University, Germany, and the other from the National Polytechnic Institute of Lorraine European School for Materials Science and Engineering, Germany. He earned his $\mathrm{PhD}$ degree from Saarland University in collaboration with the Max Planck Institute for Metals Research. Daniel is the editor of the second edition of WileyVCH's Handbook of Battery Materials, he has published numerous journal publications, and holds several patents.

Seung Min Jane Han is an associate professor in the Graduate School of Energy, Environment, Water, and Sustainability

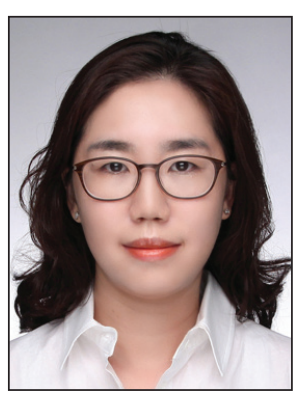
at the Korea Advanced Institute of Scienceand Technology (KAIST). She obtained her $\mathrm{PhD}$ degree in materials science and engineering in 2006 at Stanford University, USA, and served as an acting assistant professor at Stanford before joining KAIST in 2010. Her research group focuses on understanding the mechanical properties and deformation mechanisms of the nanoscale structures at a fundamental level using ex situ and in situ scanning electron microscopy/transmission electron microscopy nanoindentation methods, and applying the knowledge to evaluate and enhance the mechanical reliability of nanostructures used for different engineering applications. One specific area of her expertise is in the high strength, fatigue tolerant, metal-graphene nanolayered composite for stretchable interconnect applications. Han has been involved in the organization of several international conferences on mechanical behavior of materials, including the 2017 MRS Spring Meeting symposia on nanostructured composites.

Gabriel Montaño is a professor of chemistry and biochemistry at Northern Arizona University (NAU) and the director

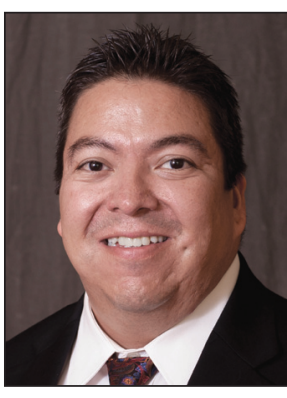
of an Advanced Functional Materials Center currently under design at NAU. He is also a distinguished scientist affiliate at the Los Alamos National Laboratory Center for Integrated Nanotechnologies, where he previously worked as a technical staff member prior to joining the NAU faculty in 2017. He received his PhD degree from Arizona State University, USA, in 2002 in the lab of Robert (Bob) Blankenship. At NAU, Montaño's group investigates membrane biophysics, in particular, supramolecular structure/function relationships at membrane interfaces in biological and biosynthetic systems. Montaño is also devoted to increasing diversity in STEM fields. He served as the president of the Society for the Advancement of Chicanos/ Hispanics and Native Americans in Science (SACNAS) from 2015 to 2016 and as a SACNAS Board of Directors member from 2011 to 2014 . He is a member of the Minority Affairs Committee of the Biophysical Society and the Hispanic Association of Colleges and Universities STEM task force.

Requests for instructions on submitting proposals for MRS Bulletin theme topics can be emailed to bulletin@mrs.org. 